Conference Paper

\title{
Self-tuning speed controller with load parameters observer for servo drives
}

Kotelnikova, A., Dmitriev, A., Vagapov, Y., Lashkevich, M., Kuraev, N., and Anuchin, A.

This is a paper presented at the 28th Int. Workshop on Electric Drives, Moscow, Russia, 27-29 Jan 2021.

Copyright of the author(s). Reproduced here with their permission and the permission of the conference organisers.

\section{Recommended citation:}

Kotelnikova, A., Dmitriev, A., Vagapov, Y., Lashkevich, M., Kuraev, N., and Anuchin, .A. (2021) 'Selftuning speed controller with load parameters observer for servo drives'. In: Proc. 28th Int. Workshop on Electric Drives, Moscow, Russia, 27-29 Jan 2021, pp. 1-5. doi: 10.1109/IWED52055.2021.9376384 


\section{Self-tuning Speed Controller with Load Parameters Observer for Servo Drives}

\author{
Anastasia Kotelnikova \\ Department of Electric Drives \\ Moscow Power Engineering Institute \\ Moscow, Russia \\ kotelnikowa-nas@yandex.ru \\ Maxim Lashkevich \\ Department of Electric Drives \\ Moscow Power Engineering Institute \\ Moscow, Russia \\ MaxSoftPage@yandex.ru
}

\author{
Alexey Dmitriev \\ Department of Electric Drives \\ Moscow Power Engineering Institute \\ Moscow, Russia \\ alexeydmitrievmp@yandex.ru \\ Nikolay Kuraev \\ Department of Electric Drives \\ Moscow Power Engineering Institute \\ Moscow, Russia \\ KurayevNM@mpei.ru
}

\author{
Yuriy Vagapov \\ Faculty of Art, Science and Technology \\ Wrexham Glyndwr University \\ Wrexham, UK \\ y.vagapov@glyndwr.ac.uk \\ Alecksey Anuchin \\ Department of Electric Drives \\ Moscow Power Engineering Institute \\ Moscow, Russia \\ anuchin.alecksey@gmail.com
}

\begin{abstract}
Servo drives are widely employed in a large range of industrial applications where the load inertia and torque are varying. This paper proposes a control approach utilizing the load parameters adaptive observers to provide a stable servo drive operation under mechanical parameters variation. In contrast to other solutions, the observers are processing real speed and acceleration values instead of their command. It was shown that the observers are capable of estimating the load torque and total inertia of the drive system under different types of commands. The simulation of the proposed control approach demonstrated that the proportional gain of the speed controller tunes automatically according to the estimated inertia ensuring the required quality of the transient regardless variation of the mechanical subsystem parameters.
\end{abstract}

Keywords-adaptive observer, servomotors, torque measurement.

\section{INTRODUCTION}

Servo motors are usually used in industrial applications required high-precision control of speed and shaft position. These systems often operate under fluctuating load conditions where the load inertia and torque are varying (for example, a robot arm manipulating the objects having different masses). Such fluctuations can significantly impact overall system performance and reduce the accuracy of speed control and shaft positioning. In order to minimize the influence of changing factors on the system operation, the dynamic load parameters have to be determined and included in the control algorithm processing.

A significant number of studies were conducted focusing on the identification of load inertia and torque for the purpose of drives control tuning. Zhao et al. [1] proposed a feed-forward control strategy, which requires the estimated total inertia, load torque and friction coefficient to be implemented into the process of adaptation of the drive control. Although this approach shows improvement in the drive performance, it results in an unacceptably large number of coefficients that have to be adjusted by many trials and errors iterations. Another research [2] suggests an original control approach integrating the inertia and torque observers, but the corresponding adaptation method remained unstudied. A simple and well-described method of mechanical parameters' identification was proposed by Kwon et al. [3]. However, the paper does not detail how this control technique can provide the required accuracy results of the parameters' identification if the load characteristics are varying during the drive operation. Different control approaches comprising the load parameters' identification are reported in other studies. For example, [4], [5] proposed control systems based on recursive least-square (RLS) observer, whereas a neural network was suggested in [6], and a variable structure control was introduced in [7].

Some of these studies have two major drawbacks. Firstly, there are disturbances in the system that are caused by the operation of an encoder. This noise influences the accuracy of parameters estimation; hence, it should be considered while modeling the system. Secondly, these methods are based on a model that uses acceleration command instead of actual motor acceleration for inertia estimation. Although this approach allows preventing inaccuracy during inertia estimation, it results in a long estimation time and makes the observer operation inefficient under particular types of commands. Also, some mathematical equations in these studies are unclear and, therefore, difficult to analyze.

This study provides an investigation of a simple adaptive controller utilizing the load parameters estimated by the observers. Each observer is processing the actual values of speed and acceleration instead of their commands. This method reduces estimation time but requires filtering of the data. The model of the proposed controller was tested under different rates of load variation and various types of position (or speed) command profiles.

\section{OBSERVER DESIGN AND MODELING}

\section{A. Electromechanical Subsystem}

Servo drives can utilize different types of motors, e.g. synchronous or induction drives. Under field-oriented control, any motor can be replaced with a first-order lag, which produces a command torque. To simplify the proposed approach analysis, a DC motor control system has been selected for the modeling.

The block diagram of the DC motor control system is shown in Fig. 1. Since the mechanical time constant is much larger than the electrical one, the system under consideration can be represented as:

$$
T_{m}-T_{L}=J \varepsilon
$$




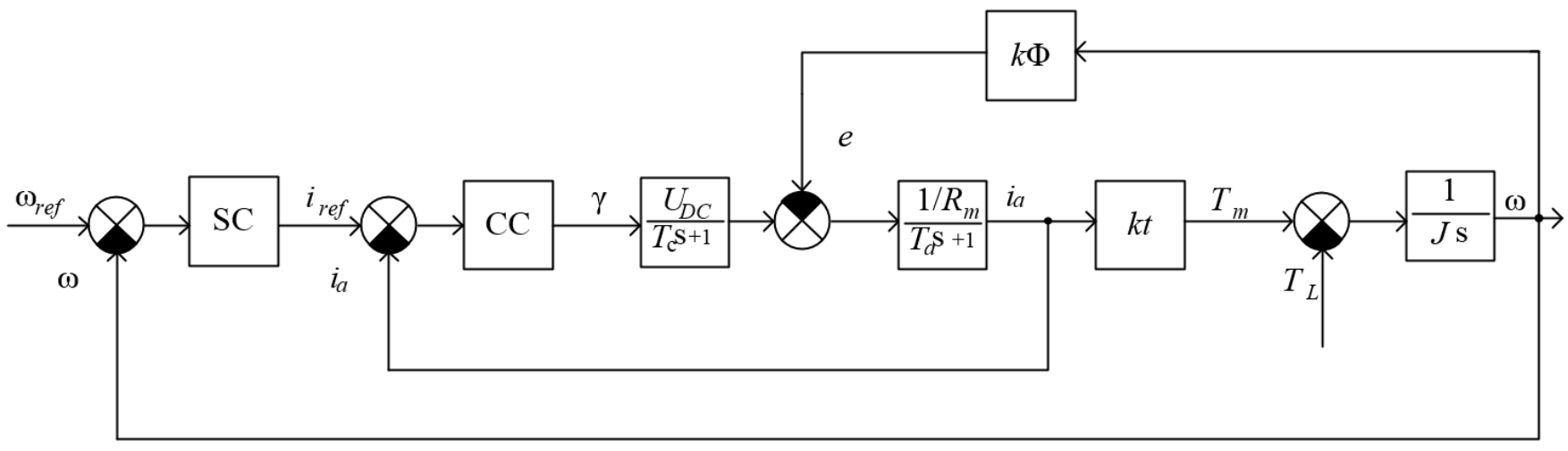

Fig. 1. DC motor and its nested loop control system.

where $T_{m}$ is the motor torque, $T_{L}$ is the load torque, $J$ is the total system inertia and $\varepsilon$ is the motor acceleration.

Fig. 1 shows a DC motor and its nested loop control system, where $e$ is back-EMF, $k \Phi$ is a back-EMF constant which in case of DC motor is equal to $k_{t}$-torque constant, CC is current controller, and SC is speed controller.

\section{B. Inertia Estimation}

Typical control system often lacks the actual values of load torque and total inertia. In addition to that, these parameters can be changed during the motor operation. Most of the time, the only information available is the rated parameters such as the motor inertia. Then (1) can be rewritten as:

$$
T_{m}=J_{e} \varepsilon+T_{L e}=J_{e} \varepsilon+T_{L}+T_{e r r},
$$

where $J_{e}$ is an estimated inertia, $T_{L e}$ is an estimated load torque $T_{e r r}$ is a torque error caused by inertia mismatch. From (2), it is evident that $J_{e}$ can be calculated if $T_{L}$ and $T_{e r r}$ are measured.

For ease of reading, this paper does not provide the derivation of the adaptation law for inertia identification. It is given as:

$$
\begin{gathered}
J_{e}=\frac{T_{e r r} \varepsilon k_{j}}{s}+J_{0}, \\
T_{e r r}=T_{\text {Lerr }}-T_{\text {Lact }},
\end{gathered}
$$

where $k_{j}$ is an adaptation rate, $J_{0}$ is the initial value of inertia, $T_{\text {Lact }}$ is the actual load torque, $T_{\text {Lerr }}$ is the load torque that includes an error in inertia estimation and $s$ is a derivative. Fig. 2 shows the block diagram of the proposed observer.

From (3) and (4) it can be concluded that inertia identification depends on both acceleration and torque error.

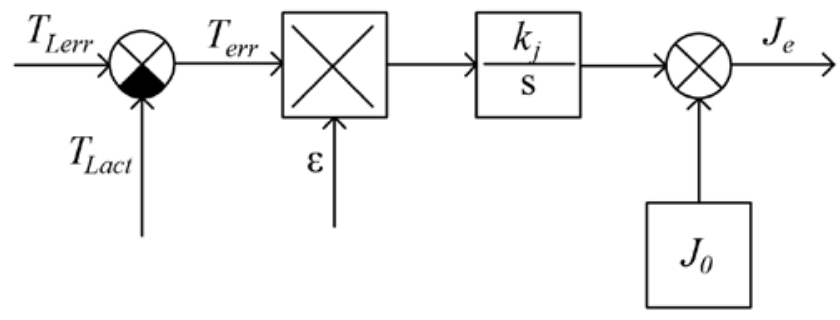

\section{Torque Error Observer}

According to the nested control method, the motor torque reference is formed by the speed loop. In order to obtain a torque that contains the inertia identification error, the same speed controller can be used. It has the actual rotor speed as a reference signal and estimated speed as a feedback signal. The controller output is the required load torque $T_{\text {Lerr }}$ that consists of both the actual load torque and the inertia estimation error. This signal provides the information to the inertia observer that indicates whether inertia must be increased or decreased. The model of this observer is shown in Fig. 3, where $\omega$ is the actual motor speed, $\omega_{\text {est }}$ is the estimated speed, SC is the same speed controller which is used in the drive control system, and $T_{m}$ is the motor torque. The $T_{\text {Lerr }}$ is used to identify the inertia. When the inertia is identified correctly, $T_{\text {Lerr }}$ becomes equal to the actual load torque $T_{\text {Lact }}$.

\section{Load Torque Identification}

Inertia observer requires the motor acceleration and the actual load torque. Equation (1) shows that the motor torque $T_{m}$ is equal to the load torque $T_{L}$ over the time periods when the motor is operating at a constant speed. That means that the load torque can be equalized to motor torque during these time periods. The motor torque can have various noises which can increase dramatically just before the motor begins to accelerate. This can lead to a wrong load torque identification. In order to avoid this problem, a low-pass filter with a high time constant can be used. In this case, peaks in the motor torque will not affect the load torque and it will be identified correctly.

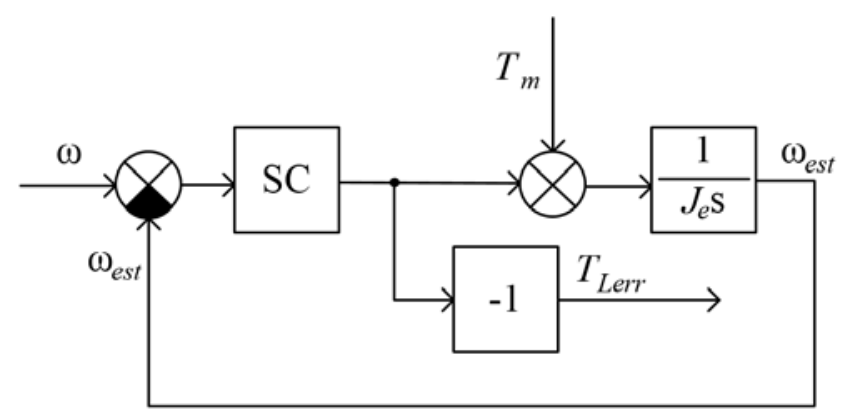

Fig. 3. Load torque and speed observer scheme (SC is a speed controller).

Fig. 2. Inertia observer block diagram. 


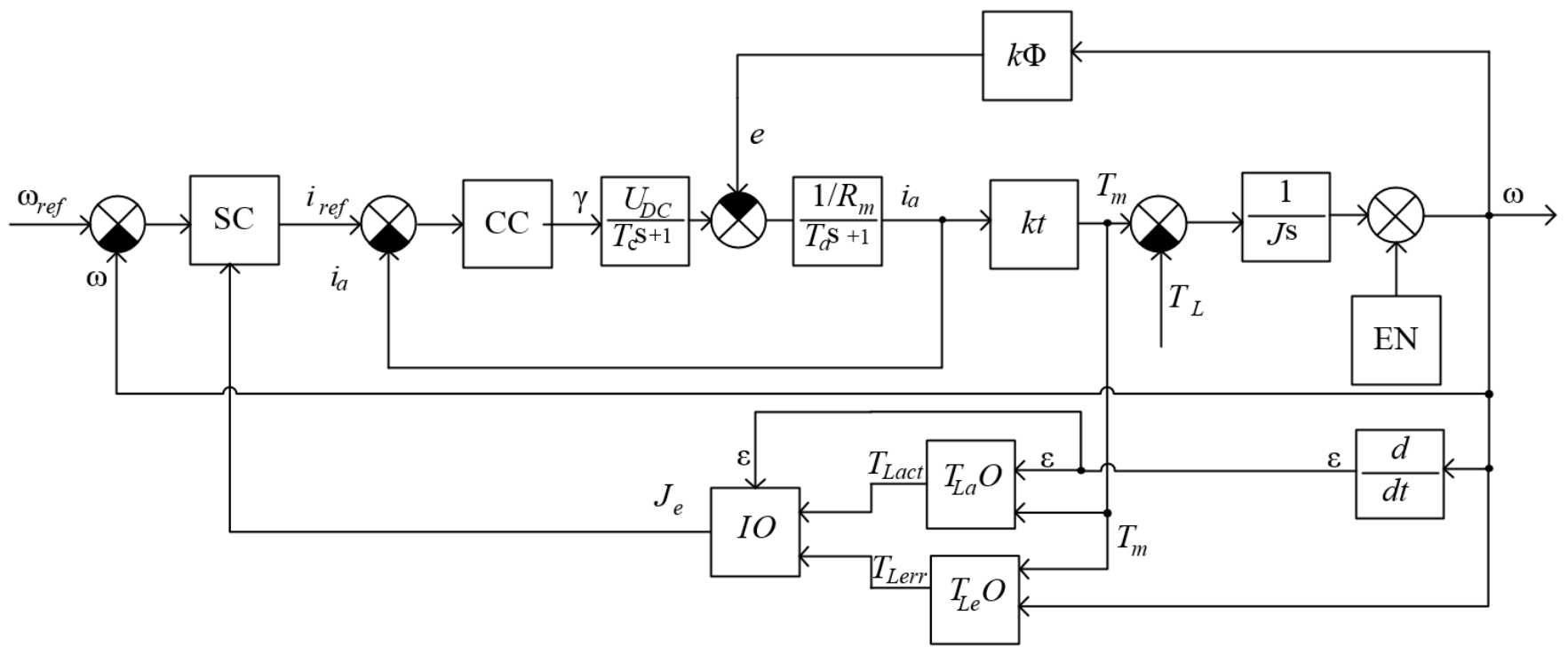

Fig. 4. System with observers and adaptation mechanism (IO-inertia observer, SC-speed controller, CC-current controller, EN-encoder noise)

\section{E. Speed Controller Tuning}

In general, the proportional gains of the speed controller are in direct proportion to the inertia of the system. The feedforward control scheme proposed in [1] has many coefficients which could be difficult to calculate. Thus, the proposed system is more robust. The system proportional gain for a DC motor control can be calculated as follows:

$$
K_{p}=\frac{J_{e s t}}{4 k \Phi T_{c}}
$$

where $T_{c}$ is the converter time constant.

The speed controller proportional gain is tuned according to the calculated inertia. It may be limited by some value of $K_{P \max }$ as the higher gain makes the system unstable.

\section{Modeling Results}

The parameters of the DC motor system used in the simulation are shown in Table I. Simulation was performed in MATLAB/Simulink.

In order to test the implemented models, the system under different rates of loads and commands was examined, and all possible advantages and drawbacks were outlined. For the test purpose, the system with adaptation algorithm was build as shown in Fig. 4, where IO is inertia observer,

TABLE I. MAIN PARAMETERS OF DC MOTOR SYSTEM

\begin{tabular}{|l|l|}
\hline \multicolumn{1}{|c|}{ Parameter } & \multicolumn{1}{c|}{ Value } \\
\hline Rated voltage & $110 \mathrm{~V}$ \\
\hline Rated current & $10 \mathrm{~A}$ \\
\hline Rated speed & $110 \mathrm{rad} / \mathrm{s}$ \\
\hline Rated back-EMF constant & $1 \mathrm{~V}^{*} \mathrm{~s} / \mathrm{rad}$ \\
\hline Rated inertia & $0.02 \mathrm{~kg} * \mathrm{~m} 2$ \\
\hline Armature resistance & $1 \mathrm{Ohm}$ \\
\hline Armature inductance & $0.05 \mathrm{H}$ \\
\hline Converter time constant & $0.001 \mathrm{~s}$ \\
\hline
\end{tabular}

EN is encoder noise represented as white noise with the sampling ratio of about 1-2 PWM cycles and variation in the range $\pm 1.5 \mathrm{rad} / \mathrm{s}$ with $95 \%$ probability, $\mathrm{T}_{\mathrm{La}} \mathrm{O}$ is the actual load torque observer, $\mathrm{T}_{\mathrm{Le}} \mathrm{O}$ is the observer of load torque that consists of both the actual load torque and the inertia estimation error.

Following the fact that all mechanical parameters of the load may be varying at the same time, the case of changing load torque and inertia was modeled. Fig. 5 shows the speed command, the estimated speed and the actual speed of the motor; the estimation of two types of the load torque (as discussed in sections $C$ and $D$ ), the estimated total inertia of the system when the real inertia is constant at the initial moment of time and then grows constantly.

In order to test the quality of estimation of all load parameters under the condition when only one value is varying, the system affected by the load torque fluctuation was examined. Fig. 6 shows the results of the estimation of all kinds of the load torque, the system inertia and the speed.

It can be seen that in both cases all observers estimated all required parameters and provided information which is in agreement with the actual values of all load characteristics. Hence, the first significant advantage of the implemented system is independence from a waveform of position (speed) command. Also, the system tracks any parameter varying over the operation time and, in any considered cases, all parameters were estimated. Another benefit of the proposed model is the simplicity of implementation of the adaptation method.

However, it should be noted that the introduced system has some disadvantages related to the adaptation ratio of the inertia observer. This coefficient requires a highly accurate tuning; otherwise, it can result in the system being unstable and results being obtained incorrectly. In addition to that, the estimated load torque should be filtered in order to prevent inaccurate inertia identification.

In order to analyze the quality of speed tracking, two cases were modeled. The first, the proportional gain of the speed controller adjusted to the system`s inertia and the second, it did not change when the total system`s inertia was 


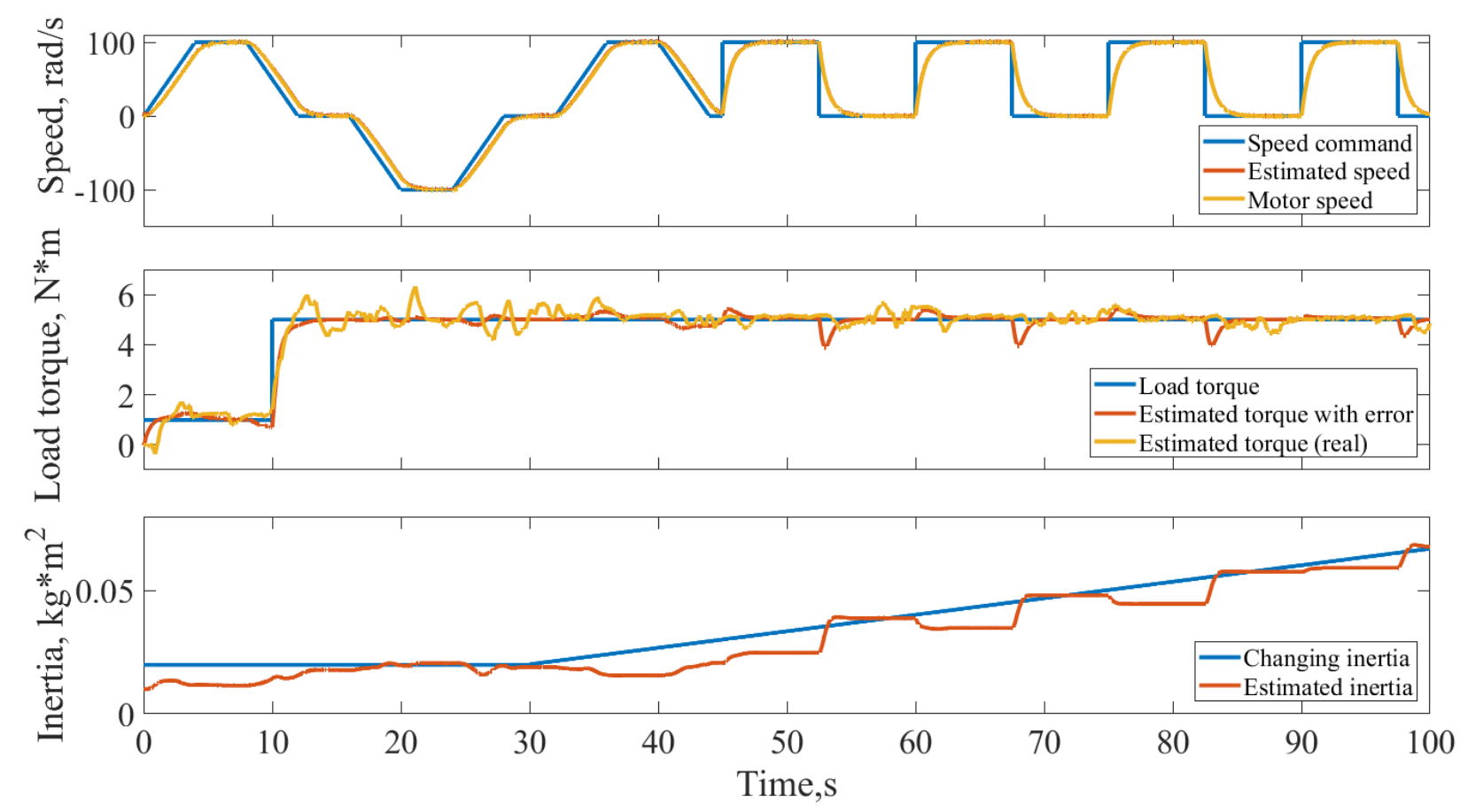

Fig. 5. Parameters' references and their estimation under the varying condition of all mechanical parameters; load torque LPF constant is $1 \mathrm{~s}$

varying. Fig.7 shows the result of the estimation of the total inertia and the speed error in both cases. It can be seen that when the speed controller tunes according to the new system`s parameters, the error decreases.

\section{CONCLUSION}

To minimize the impact of the changing load parameters on the stability of a servo drive operation, the identification and adaptation methods were proposed and discussed. The suggested observers are capable of estimating the load inertia and torque. The adaptation algorithms consist of a simple self-adjustment of the speed controller proportional gain according to the calculated inertia. The system can operate under different load rates and waveforms of the speed command. The proposed system modeling demonstrated that it operates effectively and stable even under the noise condition occurred in the controller. Speed-tracking error decreases when the speed controller tunes according to the estimated inertia.

Future work will be devoted to the experimental verification of the proposed method using a servomotor and modification of the observer for the operation with a twomass system.
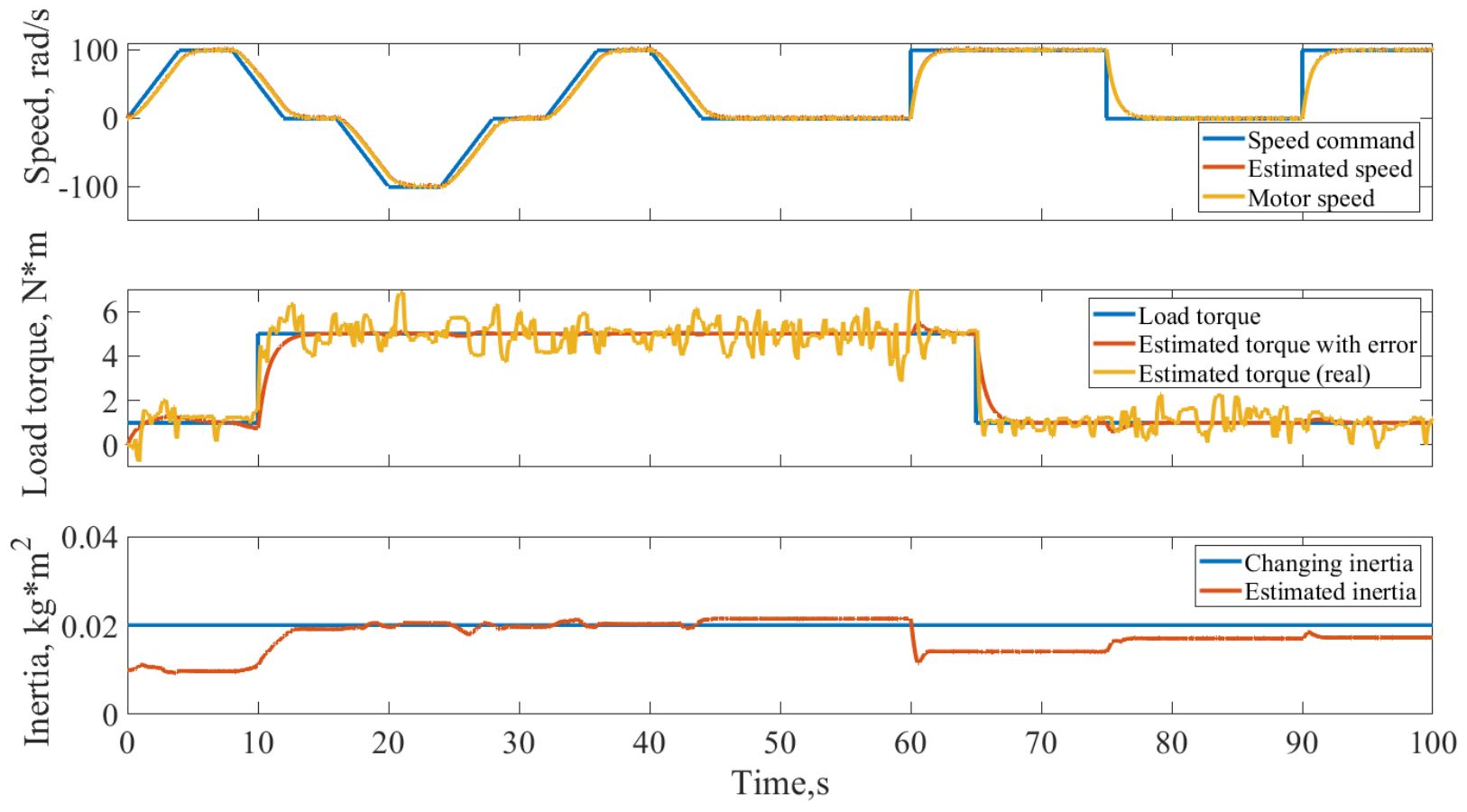

Fig. 6. Estimated load torque at constant inertia; estimated load torque LPF constant is $1 \mathrm{~s}$, estimated load torque with error LPF constant is $0.2 \mathrm{~s}$ 

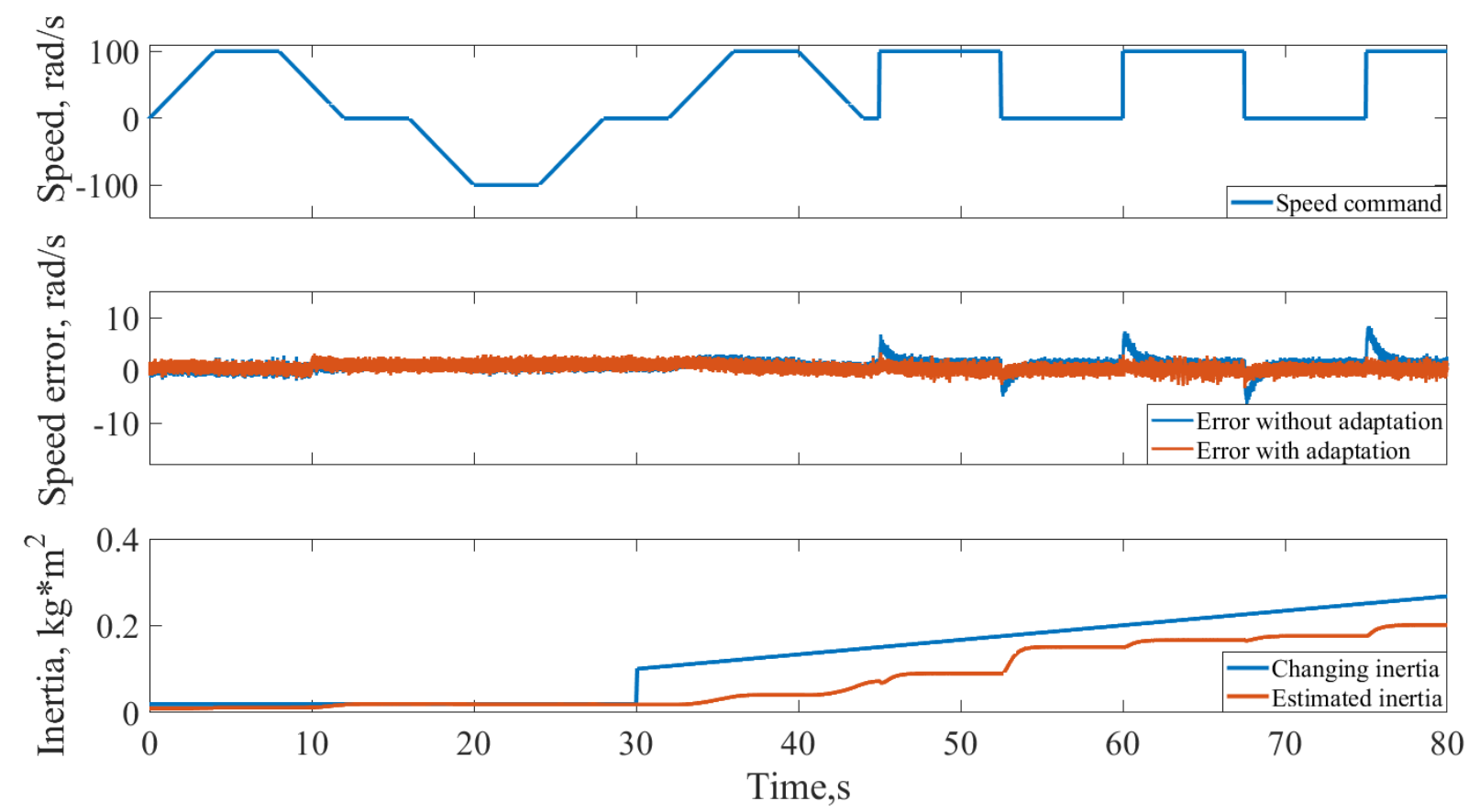

Fig. 7. Speed command, system`s inertia and speed error in two case

\section{REFERENCES}

[1] S. Zhao, Y. Chen, and Y. Mao, "Adaptive load observer-based feedforward control in PMSM drive system,” Transactions of the Institute of Measurement and Control, vol. 37, no. 3, pp 414-424, 2015.

[2] S. Yang, and J. Lin, “Observer-based automatic control loop tuning for servo motor drives," in Proc. 10th IEEE Int. Conf. on Power Electronics and Drive Systems (PEDS), Kitakyushu, Japan, 22-25 April 2013, pp. 302-305.

[3] T. Kwon, S. Sul, H. Nakamura, and K. Tsuruta, "Identification of the mechanical parameters for servo drive," in Proc. IEEE Industry Applications Conf. 41st IAS Annual Meeting, Tampa, FL, USA, 8-12 Oct. 2006, pp. 905-910.

[4] X. Cao, and L. Fan, "Self-tuning PI controller for permanent magnet synchronous motor based on iterative learning control," in Proc. 2nd
Int. Symp. on Intelligent Information Technology Application, Shanghai, China, 20-22 Dec. 2008, pp. 756-760.

[5] M. De Campos, E. G. Caratti, and H. A. Grundling, "Design of a position servo with induction motor using self-tuning regulator and Kalman filter,” in Proc IEEE Industry Applications Conf. 35th IAS Annual Meeting and World Conf. on Industrial Applications of Electrical Energy, Rome, Italy, 8-12 Oct. 2000, vol. 3, pp. 16131618.

[6] H. Li, and Q. Wang, "Self-tuning controller for servo motor with an adaptive disturbance observer," in Proc. 2nd Int. Conf. on Advanced Computer Control, Shenyang, China, 27-29 March 2010, pp. 277281.

[7] W. Bahn, D. Dan Cho, S. Lee, and S. Lee, "Tuningless servo controller using variable structure control and disturbance compensation,” In Proc. IEEE Int. Conf. on Industrial Technology (ICIT), Busan, South Korea, 26 Feb.-1 March 2014, pp. 96-99. 